\title{
Barrier function and microbiotic dysbiosis in atopic dermatitis
}

This article was published in the following Dove Press journal:

Clinical, Cosmetic and Investigational Dermatology

15 September 2015

Number of times this article has been viewed

\author{
Sophie Seite' \\ Thomas Bieber ${ }^{2}$ \\ 'La Roche-Posay Dermatological \\ Laboratories, Asnières, France; \\ ${ }^{2}$ Department of Dermatology and \\ Allergy, Friedrich-Wilhelms-University, \\ Bonn, Germany
}

\begin{abstract}
Atopic dermatitis (AD) or atopic eczema is the common inflammatory skin disorder, the prevalence of which has considerably increased during the last 30 years. It affects $15 \%-30 \%$ of children and $2 \%-10 \%$ of adults. AD characteristically alternates between periods of exacerbation or flares and periods of remission, which may be therapeutically induced or spontaneous. Current knowledge about AD includes abnormalities of the skin barrier (physical and chemical), the immune barrier, and more recently, the microbial barrier or microbiota. There is growing evidence for a tight relationship between them. To obtain satisfactory control of this condition, the clinical strategy to manage $\mathrm{AD}$ involves prescribing both anti-inflammatory medications and dermocosmetic products. The role of the physician is therefore to advise the patient with regard to hygiene measures aimed to help to improve these three barriers or to prevent any further deterioration.
\end{abstract}

Keywords: atopic dermatitis, eczema, skin barrier, microbiome, immunity, dermocosmetic

\section{Introduction}

Atopic dermatitis (AD) or atopic eczema is the common inflammatory skin disorder with a substantial socioeconomic burden and impact on quality of life. ${ }^{1} \mathrm{AD}$ is considered as the first step of the so-called atopic march. ${ }^{2}$ The three main pillars of the management of AD, which are the basis of most guidelines include 1) an allergological work-up and identification of provocation factors, 2) a severity-adapted prescription of anti-inflammatory medications, and 3) a basic therapy, ie, an ideally individually adapted skin care aimed to improve the impaired barrier function. ${ }^{3,4} \mathrm{AD}$ was initially thought to be an immunological disease, where the chronic inflammation of the skin causes and/or exacerbates the skin barrier impairment. The pathophysiology of AD is as complex ${ }^{5}$ as the clinical phenotype, and recent studies revealed the genetic origin of skin barrier function abnormalities and the role of filaggrin mutations in $10 \%-30 \%$ of patients with AD in Caucasian populations. ${ }^{5,6}$ They also revealed the involvement of other stratum corneum proteins in an innate deficiency of the physical and chemical skin barriers. ${ }^{7,8}$ These abnormalities enable different environmental factors to penetrate the epidermis, including chemical, allergic, or infectious agents. This results in an inappropriate immune response with chronic inflammation and the presence of allergens or pathogenic agents, which could stimulate a T-helper 2/T-helper 22-type reaction. This inflammatory reaction can itself impact on the integrity of the barrier function. Finally, a kind of selective antimicrobial peptide deficiency leads to a loss of microbial diversity with an overabundance of the Staphylococcus bacterial
Correspondence: Sophie Seite

La Roche-Posay Dermatological Laboratories, II 0 Avenue Henri Barbusse, 92602 Asnières Cedex, France

Tel +33 ol 46886544

Fax +33 0I 46886688

Email sophie.seite@loreal.com 
genus, witnessing the role of the microbiome in the pathogenesis of AD. ${ }^{9}$ This abnormal microbial colonization justifies $\mathrm{AD}$ being regarded as a state of dysbiosis.

The overall therapeutic strategy used in mild-to-moderate AD management aims to rapidly reduce the inflammatory response and/or regulate the immune response with either topical corticosteroids or calcineurin inhibitors ${ }^{10}$ and to improve the skin barrier function using emollients to rebuild the chemical and/or physical lipid barrier. ${ }^{11}$ Basic therapy improving the barrier function is also an established part of the management of severe forms where systemic treatments have to be considered. ${ }^{12-14}$ Moreover, direct manipulation of the cutaneous microbiome to treat the dysbiosis and reestablish the microbial diversity will represent a new treatment approach to be considered in combination with current strategies. Advice provided by the physician is essential. In daily practice, the practitioner's attitude is to concomitantly prescribe medications and skin care products belonging to dermocosmetics. This approach forms part of a genuine care protocol where each product has its place, from daily hygiene to medical treatments, to ensure a complete and personalized approach. The skin is an organ, which must be treated in its entirety in order to obtain both a therapeutic result and the patient's psychological and esthetic satisfaction.

\section{Restoring the skin barrier in AD using emollients}

Emollients are prescribed to improve the skin barrier function. They restore stratum corneum lipids and may have anti-inflammatory properties, which are demonstrated in in vitro or in vivo clinical trials. ${ }^{15}$ As it is vital for atopic patients to limit moisture loss and restore the hydrolipidic film, emollients are an essential part of their daily skin care regimen. "To moisturize" does not only mean providing moisture, it also means preventing moisture evaporation from the skin.

To meet these needs emollients can be formulated with the following ingredients:

1. Emollient agents make the skin softer and more pliable by filling the space between corneocytes and restoring the physical barrier function. Raw materials with this property include 1) hydrogenated or nonhydrogenated vegetable oils, 2) mineral oils (eg, paraffin and petroleum jelly), 3) vegetable butters (eg, shea and cocoa), 4) alcohols, fatty acids, and esters, 5) triglycerides, and 6) ceramides.

2. Humectant or moisturizing agents are water-soluble substances, which help the stratum corneum capture water from the outside and rebalance the cutaneous hydrolipidic film. Glycerol (and glycerol derivatives), urea, lactic acid, and $\alpha$ - and $\beta$-hydoxyacids are the most commonly used humectant ingredients.

3. Occlusive agents create a sealed barrier and prevent moisture evaporation from the surface of the epidermis. Petroleum jelly is undoubtedly the most effective occlusive substance but agents with film-forming properties also exist such as lipophilic (silicones and silicone elastomers) and hydrophilic film-forming agents. The latter consist of very large molecules capable of "capturing" water and forming a film on the surface of the skin stopping water evaporation. They include 1) proteins (collagen), 2) complex carbohydrates (glycosaminoglycan: hyaluronic acid and sulfated sugars), 3) synthetic polymers (carbomer, 2-acrylamido-2-methylpropane sulfonic acid $\left[\mathrm{AMPS}^{\circledR}\right]$, and 4) waxes. However, the disadvantage of these occlusive agents is their sticky and/or greasy consistency making them less pleasant to use.

The quality of the emollient is best when a perfect balance is obtained from combining 1) efficacy, 2) cosmetic quality, and 3) patient tolerance. This is the art of formulation. The patient's compliance is highly dependent on the formulation. ${ }^{16}$ The formulation must ideally meet dermatologist expectations as well as patient needs and must be effective. Different formulations have different objectives; some ingredients have moisturizing efficacy (eg, glycerin and pyroglutamic acid), whereas others promote penetration of active ingredients into the skin, ensuring their bioavailability. Therefore, emollients can contribute to the success of dermatological treatments by ensuring optimal hygiene and relieving the irritation and dryness caused either by the condition or by the medical treatments. Some emollients can also provide real clinical benefit, alone or in combination with medical products. ${ }^{17}$

Numerous formulations can be used; however for a condition like $\mathrm{AD}$, the most appropriate pharmaceutical formula contains raw materials that provide a therapeutic effect, with few side effects, especially with no sensitization risk. Raw materials are chosen based on the following criteria: 1) safety, 2) purity, and 3) cosmetovigilance data. Any known allergens are avoided, and the packaging is designed to limit the use of preservatives (eg, fine nozzle tubes that can reduce microbiological contamination, or single doses, which ensure a sterile cosmetic product). Water content provides an environment suited for bacterial growth, so nowadays, formulation chemists tend to combine raw materials with low free water content, thus allowing to reduce the preservative concentration as much as possible. 


\section{Manipulation of the cutaneous microbiota in AD by emollients}

For decades, microbiologists throughout the world have attempted to improve bacterial culture media in laboratory. From a bacterial point of view, skin is a culture medium. On average, skin houses one million bacteria per centimeter square. This specific ecosystem is poorly understood. Cosmetic and dermatological treatments provide the skin with external components thus modifying its nutritional characteristics and acting on its microbiome. This million bacteria per centimeter square is represented by several hundred different microbes that cohabit, fight, and collaborate the image of life in a human community. ${ }^{18,19}$

For a long time, it has been known that Staphylococcus aureus is present on both healthy and affected skin of $>90 \%$ of patients with $\mathrm{AD}$. The presence of $S$. aureus is at least partially explained by an antimicrobial peptide deficiency and by the poor quality of the skin barrier, which allows easy access to complex substrates..$^{20,21}$ The arrival of metagenomics enabled scientists to study the cutaneous microbiome more precisely, particularly in skin affected by AD. ${ }^{9,22}$ These studies showed that in $\mathrm{AD}$ there is a loss of microbial diversity. During flares, this loss of diversity and the predominance of Staphylococci worsen in correlation with flare severity. Bacterial diversity is greater when treatment is proactive compared to no treatment. This suggests that there is a relationship between the recovery of bacterial diversity and remission. ${ }^{9}$ Additionally, the presence of Staphylococcus epidermidis also increases during flares, which could correspond to a compensatory and antagonistic mechanism in response to the increase of $S$. aureus. ${ }^{23}$

Given that each individual has a unique microbiome linked with his/her genetic make-up, diet, lifestyle, and surrounding environment and that the majority of studies performed in this field compare healthy to atopic individuals, it is important to validate the observations made in these studies by comparing intrapatient skin areas. ${ }^{24}$ Recent studies have confirmed that AD lesions are dominated by Staphylococci and have a lower microbial diversity than that of the unaffected adjacent skin. ${ }^{9,23}$ An emollient can equalize the composition of an affected or lesional area (AF) to a state similar to that of uninvolved skin (nonlesional or unaffected UAF area) (Figures 1 and 2). ${ }^{23}$ Also keratolytic bacteria (ie, Xanthomonadaceae family), naturally present on the skin, have access to keratin, which is favorable to their growth. ${ }^{23,25}$ These results reveal new insight into the cutaneous microbiome, the targeted manipulation of which is a new approach for $\mathrm{AD}$ treatment.
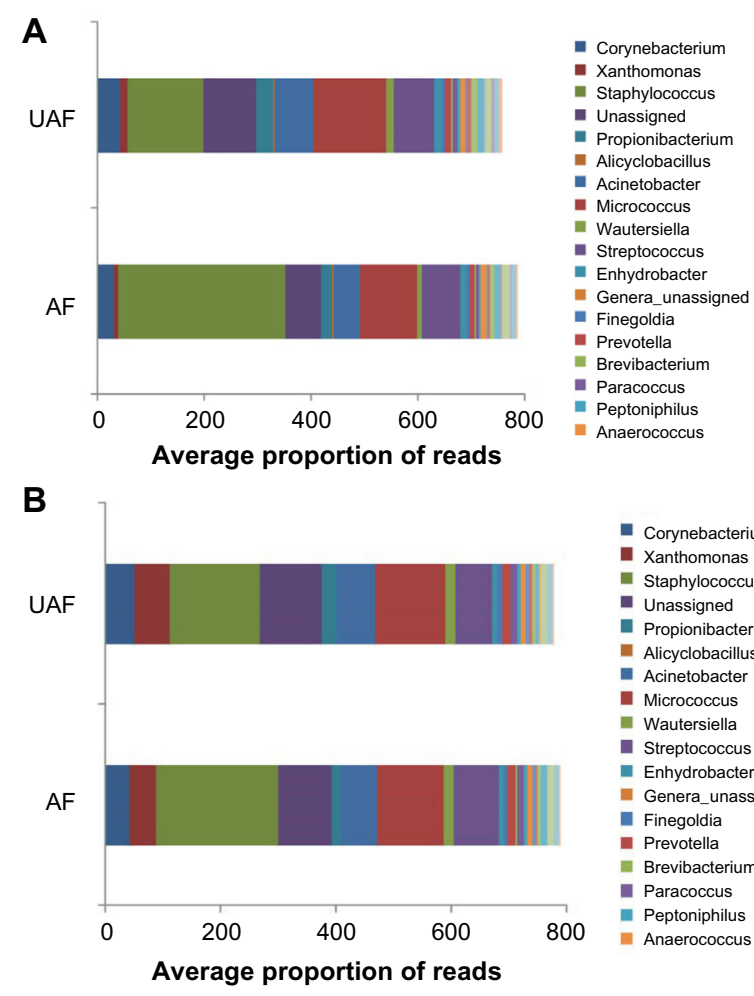

- Corynebacterium

- Xanthomonas

- Staphylococcus

- Unassigned

- Propionibacterium

- Alicyclobacillus

- Acinetobacter

- Micrococcus

Wautersiella

- Streptococcus

- Enhydrobacter

- Genera_unassigned

- Finegoldia

- Prevotella

- Brevibacterium

- Paracoccus

-1 Peptoniphilus

- Anaerococcus

Figure I Bacterial landscape of the 30 main bacterial genera before (D0 - A) and after (D83 - B) 83 days of daily treatment with emollient on the affected (lesional) zones (AF) and the unaffected (nonlesional) adjacent zones of healthy appearance (UAF) in atopic patients ( $\mathrm{N}=49$ ).

Notes: The bacterial landscape of the atopic lesions before treatment is dominated by Staphylococci. Swabs were taken in axenic conditions, in order to ensure that only skin flora is collected, from affected (AF) and proximal unaffected skin (UAF) at D0 and D83, and the I6S ribosomal RNA bacterial gene was used to analyze the composition of bacterial communities as previously described. $X$-axis corresponding to the average proportion of reads for each genus.

Abbreviations: UAF, unaffected; $A F$, affected; $D$, day.

Other than in cases of superinfection, combating S. aureus has never been definitely proven to be of therapeutic benefit. Topical antiseptic or antibiotic treatments, for example, the use of diluted bleach baths, are still sometimes recommended, despite this lack of demonstrated efficacy on the microbiome, their potentially irritant effect, the risk of bacterial resistance induction, and the action on bacteria, which could control S. aureus such as $S$. epidermidis. ${ }^{26}$

Numerous clinical trials have been conducted with the objective of demonstrating a preventive action of probiotic supplementation (living nonpathogenic bacteria) on the initial development and/or flares and severity of AD. Lastly, several meta-analyses support the conclusion that prebiotics or probiotics could have a preventive effect on the development of AD but do not appear to have any direct therapeutic benefit. ${ }^{27-29}$ Nevertheless, some recent studies would disagree with this conclusion. ${ }^{30-32}$

Similarly, applying dead extracts from nonpathogenic bacteria onto the skin could be a new therapeutic approach 


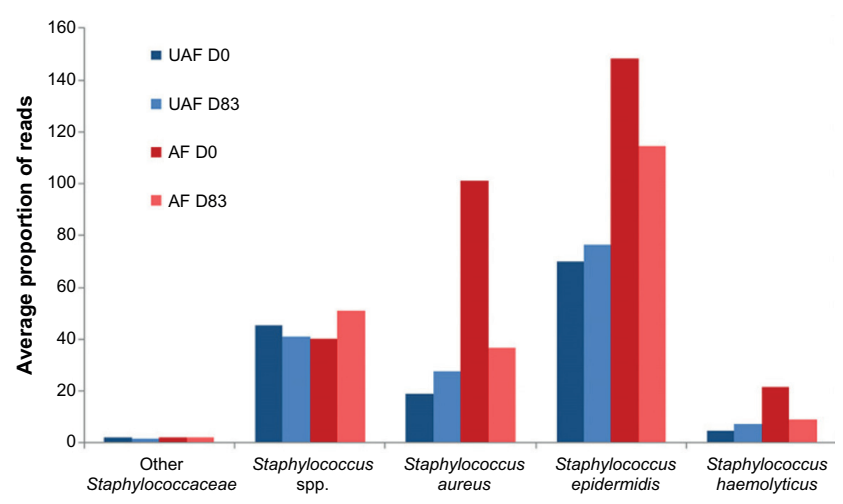

Figure 2 Different species of Staphylococci present on the affected zones (AF - in red) and the unaffected zones (UAF - in blue) of atopic patients $(\mathrm{N}=49)$ before (D0, darker) and after (D83, lighter) 83 days of daily treatment with an emollient.

Notes: Staphylococcus epidermidis, Staphylococcus aureus, and Staphylococcus haemolyticus are overabundant on the atopic lesions before treatment and are reduced after treatment. $Y$-axis corresponding to the average proportion of reads for each species.

Abbreviations: UAF, unaffected; AF, affected; D, day.

to modulate or balance the immune system and would enable manipulation of the cutaneous microbiome. This hypothesis is based on results obtained from the following four key studies: 1) a significant improvement in the severity of $\mathrm{AD}$ in a randomized trial using a cream containing a lysate from a Gram-negative bacterium, Vitreoscilla filiformis, in the treatment of $\mathrm{AD} ;{ }^{33}$ ) a mouse model of $\mathrm{AD}$ revealing that the V. filiformis lysate is able to reduce the clinical inflammatory manifestations and the inflammatory reaction to an allergen when it was applied, suggesting a significant modulation of the immune response; ${ }^{34} 3$ ) an in vitro model on a reconstructed epidermis revealed that, surprisingly, the V.filiformis lysate stimulated the $\beta$-defensin production and other innate immune defense mechanisms through activation of toll-like receptor $2 ;^{35}$ and finally 4) a randomized trial showing the reduction in the probability of relapse of $\mathrm{AD}$ concomitant to a substantial impact on the composition of the microbiome. ${ }^{36} \mathrm{As}$ concluded in a recent review on this topic, after identification of diminished bacterial diversity in the skin microbiome of atopic individuals with consecutive loss of anti-inflammatory and tolerogenic interleukin 10, substitution of these tolerance promoting innate immune signals using microbes or microbial components is a new and promising therapeutic strategy. ${ }^{37}$

\section{Conclusion}

To control $\mathrm{AD}$ is the goal of its management, which involves the concomitant prescription of medical products and dermocosmetic products. The role of the dermatologist is to prescribe and inform the patient about appropriate hygiene measures that restore and/or prevent worsening of the skin barrier alteration while strengthening the immune barrier and regulating the skin microbiome. A well-balanced emollient with effective ingredients that restore skin barrier function, acceptable cosmetic quality, and excellent dermatological tolerance, supplemented with ingredients able to revive natural cutaneous defenses by providing nutritional supplements to regulate the cutaneous microbiota, offers the best option for AD. Finally, an impaired skin barrier function has been suspected to be instrumental in the emergence of immunoglobulin E-mediated sensitization and the atopic march. There is first evidence that an adapted basic therapy could reduce and/or delay the appearance of $\mathrm{AD}$ in newborns at high risk to develop this condition..$^{38-40}$ Thus, emollients able to impact on the microbiotic dysbiosis could be part of the future early intervention strategies aimed to prevent $\mathrm{AD}$ and possibly the atopic march.

\section{Acknowledgments}

The authors would like to thank N Cleren and G Sore for information provided about the formulation and dosage forms of emollients and R Martin for his microbiological expertise. The authors also acknowledge the editing support of Charlotte Wright (Speak the Speech Consulting).

\section{Disclosure}

S Seité is an employee of La Roche-Posay but has no financial interest in the company. $\mathrm{T}$ Bieber has been a lecturer for $\mathrm{La}$ Roche-Posay. The authors report no other conflicts of interest in this work.

\section{References}

1. Bieber T. Atopic dermatitis. N Engl J Med. 2008;358(14):1483-1494.

2. Dharmage SC, Lowe AJ, Matheson MC, Burgess JA, Allen KJ, Abramson MJ. Atopic dermatitis and the atopic march revisited. Allergy. 2013;69:17-27.

3. Ring J, Alomar A, Bieber T, et al. Guidelines for treatment of atopic eczema (atopic dermatitis) part I. J Eur Acad Dermatol Venereol. 2012; 26(8):1045-1060.

4. Eichenfield LF, Tom WL, Chamlin SL, et al. Guidelines of care for the management of atopic dermatitis: section 1. Diagnosis and assessment of atopic dermatitis. J Am Acad Dermatol. 2014;70(2):338-351.

5. Eyerich K, Novak N. Immunology of atopic eczema: overcoming the Th1/Th2 paradigm. Allergy. 2013;68(8):974-982.

6. Smith FJ, Irvine AD, Terron-Kwiatkowski A, et al. Loss-of-function mutations in the gene encoding filaggrin cause ichthyosis vulgaris. Nat Genet. 2006;38(3):337-342.

7. Boguniewicz M, Leung DY. Atopic dermatitis: a disease of altered skin barrier and immune dysregulation. Immunol Rev. 2011;242(1):233-246.

8. Kuo IH, Yoshida T, De Benedetto A, Beck LA. The cutaneous innate immune response in patients with atopic dermatitis. J Allergy Clin Immunol. 2013;131(2):266-278.

9. Kong HH, Oh J, Deming C, et al. Temporal shifts in the skin microbiome associated with disease flares and treatment in children with atopic dermatitis. Genome Res. 2012;22(5):850-859.

10. Schmitt J, von Kobyletzki L, Svensson A, Apfelbacher C. Efficacy and tolerability of proactive treatment with topical corticosteroids and calcineurin inhibitors for atopic eczema: systematic review and meta-analysis of randomized controlled trials. Br J Dermatol. 2011;164(2):415-428. 
11. Ring J, Alomar A, Bieber T, et al. Guidelines for treatment of atopic eczema (atopic dermatitis) Part II. J Eur Acad Dermatol Venereol. 2012; 26(9):1176-1193.

12. Simon D, Bieber T. Systemic therapy for atopic dermatitis. Allergy. 2014;69(1):46-55.

13. Bieber T, Straeter B. Off-label prescriptions for atopic dermatitis in Europe. Allergy. 2015;70(1):6-11.

14. Howell MD, Parker ML, Mustelin T, Ranade K. Past, present, and future for biologic intervention in atopic dermatitis. Allergy. 2015; 70(8):887-896.

15. Lane ME, Hadgraft J, Oliveira G, Vieira R, Mohammed D, Hirata K. Rational formulation design. Int J Cosmet Sci. 2012;34(6):496-501.

16. Hon KL, Ching GK, Leung TF, Choi CY, Lee KK, Ng PC. Estimating emollient usage in patients with eczema. Clin Exp Dermatol. 2010; 35(1):22-26.

17. Dreno B, Araviiskaia E, Berardesca E, et al. The science of dermocosmetics and its role in dermatology. J Eur Acad Dermatol Venereol. 2014;28(11):1409-1417.

18. Grice EA, Kong HH, Conlan S, et al. Topographical and temporal diversity of the human skin microbiome. Science. 2009;324(5931):1190-1192.

19. Grice EA, Segre JA. The human microbiome: our second genome. Annu Rev Genomics Hum Genet. 2012;13:151-170.

20. Ong PY, Ohtake T, Brandt C, et al. Endogenous antimicrobial peptides and skin infections in atopic dermatitis. N Engl J Med. 2002;347(15) 1151-1160.

21. Nakatsuji T, Gallo RL. Antimicrobial peptides: old molecules with new ideas. J Invest Dermatol. 2012;132(3 pt 2):887-895.

22. Grice EA. The skin microbiome: potential for novel diagnostic and therapeutic approaches to cutaneous disease. Semin Cutan Med Surg 2014;33(2):98-103.

23. Seite S, Flores GE, Henley JB, et al. Microbiome of affected and unaffected skin of patients with atopic dermatitis before and after emollient treatment. J Drugs Dermatol. 2014;13(11):1365-1372.

24. Grice EA, Segre JA. The skin microbiome. Nat Rev Microbiol. 2011; 9(4):244-253.

25. Yamamura S, Morita Y, Hasan Q, Yokoyama K, Tamiya E. Keratin degradation: a cooperative action of two enzymes from Stenotrophomonas sp. Biochem Biophys Res Commun. 2002;294(5):1138-1143. [Erratum in: Biochem Biophys Res Commun. 2002;295(4):1034].

26. Huang JT, Abrams M, Tlougan B, Rademaker A, Paller AS. Treatment of Staphylococcus aureus colonization in atopic dermatitis decreases disease severity. Pediatrics. 2009;123(5):e808-e814.

27. Panduru M, Panduru NM, Salavastru CM, Tiplica GS. Probiotics and primary prevention of atopic dermatitis: a meta-analysis of randomized controlled studies. J Eur Acad Dermatol Venereol. 2015;29(2): $232-242$.
28. Muraro A, Halken S, Arshad SH, et al. EAACI food allergy and anaphylaxis guidelines. Primary prevention of food allergy. Allergy. 2014;69(5):590-601.

29. Kim SO, Ah YM, Yu YM, Choi KH, Shin WG, Lee JY. Effects of probiotics for the treatment of atopic dermatitis: a meta-analysis of randomized controlled trials. Ann Allergy Asthma Immunol. 2014;113(2): 217-226.

30. Wang IJ, Wang JY. Children with atopic dermatitis show clinical improvement after Lactobacillus exposure. Clin Exp Allergy. 2015; 45(4):779-787.

31. Drago L, De Vecchi E, Toscano M, Vassena C, Altomare G, Pigatto P. Treatment of atopic dermatitis eczema with a high concentration of Lactobacillus salivarius LS01 associated with an innovative gelling complex: a pilot study on adults. J Clin Gastroenterol. 2014;48 (Suppl 1):S47-S51.

32. Niccoli AA, Artesi AL, Candio F, et al. Preliminary results on clinical effects of probiotic Lactobacillus salivarius LS01 in children affected by atopic dermatitis. J Clin Gastroenterol. 2014;48(Suppl 1):S34-S36.

33. Gueniche A, Knaudt B, Schuck E, et al. Effects of nonpathogenic gramnegative bacterium Vitreoscilla filiformis lysate on atopic dermatitis: a prospective, randomized, double-blind, placebo-controlled clinical study. Br J Dermatol. 2008;159(6):1357-1363.

34. Volz T, Skabytska Y, Guenova E, et al. Nonpathogenic bacteria alleviating atopic dermatitis inflammation induce IL-10-producing dendritic cells and regulatory Tr1 cells. J Invest Dermatol. 2014;134(1): 96-104.

35. Mahe YF, Perez MJ, Tacheau C, et al. A new Vitreoscilla filiformis extract grown on spa water-enriched medium activates endogenous cutaneous antioxidant and antimicrobial defenses through a potential Toll-like receptor 2/protein kinase C, zeta transduction pathway. Clin Cosmet Investig Dermatol. 2013;6:191-196.

36. Seité S, Zelenkova H, Martin R, Fierer N. Using a Specific Emollient to Manage Skin Microbiome Dysbiosis. Vancouver, Canada: World Congress of Dermatology; 2015.

37. Biedermann T, Skabytska Y, Kaesler S, Volz T. Regulation of T cell immunity in atopic dermatitis by microbes: the Yin and Yang of cutaneous inflammation. Front Immunol. 2015;6:353.

38. Flohr C, Mann J. New approaches to the prevention of childhood atopic dermatitis. Allergy. 2014;69(1):56-61.

39. Simpson EL, Chalmers JR, Hanifin JM, et al. Emollient enhancement of the skin barrier from birth offers effective atopic dermatitis prevention. J Allergy Clin Immunol. 2014;134(4):818-823.

40. Fukuie T, Nomura I, Horimukai K, et al. Proactive treatment appears to decrease serum immunoglobulin-E levels in patients with severe atopic dermatitis. Br J Dermatol. 2010;163(5):1127-1129.
Clinical, Cosmetic and Investigational Dermatology

\section{Publish your work in this journal}

Clinical, Cosmetic and Investigational Dermatology is an international, peer-reviewed, open access, online journal that focuses on the latest clinical and experimental research in all aspects of skin disease and cosmetic interventions. All areas of dermatology will be covered; contributions will be welcomed from all clinicians and

\section{Dovepress}

basic science researchers globally. This journal is indexed on CAS. The manuscript management system is completely online and includes a very quick and fair peer-review system, which is all easy to use. Visit http://www.dovepress.com/testimonials.php to read real quotes from published authors. 